\title{
O.S.P.
}

L'orientation scolaire et professionnelle

$39 / 1 \mid 2010$

S'orienter : construire sa vie

\section{Le conseil constructiviste auprès d'adolescents : sa pertinence et ses limites}

Constructivist counselling for adolescents: Pertinence and limits

\section{Bernadette Dumora}

\section{(2) OpenEdition}

Journals

Édition électronique

URL : http://journals.openedition.org/osp/2489

DOI : 10.4000/osp.2489

ISSN : 2104-3795

Éditeur

Institut national d'étude du travail et d'orientation professionnelle (INETOP)

Édition imprimée

Date de publication : 15 mars 2010

Pagination : 119-136

ISSN : 0249-6739

\section{Référence électronique}

Bernadette Dumora, «Le conseil constructiviste auprès d'adolescents : sa pertinence et ses limites », L'orientation scolaire et professionnelle [En ligne], 39/1 | 2010, mis en ligne le 15 mars 2013, consulté le 16 décembre 2020. URL : http://journals.openedition.org/osp/2489; DOI : https://doi.org/10.4000/ osp.2489

Ce document a été généré automatiquement le 16 décembre 2020.

(c) Tous droits réservés 


\title{
Le conseil constructiviste auprès d'adolescents : sa pertinence et ses limites
}

Constructivist counselling for adolescents: Pertinence and limits

\author{
Bernadette Dumora
}

\section{Introduction}

1 L'article de Savickas et al. propose un renouvellement de la conception du conseil pour répondre aux besoins des personnes dans le contexte du $21^{\mathrm{e}}$ siècle: le postulat constructiviste de ce renouvellement est à l'opposé du postulat d'immanence qui soustendait les modèles traditionnels de la psychologie de l'orientation. On a en effet longtemps fonctionné dans l'orientation comme s'il y avait un «déjà-là » des préférences pour l'avenir professionnel, un « for intérieur » pulsionnel qu'il s'agirait en quelque sorte de faire émerger sous la forme idéale d'une intention ou d'un projet. La référence à l'objectif d'aide à la construction de soi renverse ce point de vue: l'intention n'est pas à découvrir mais à construire, à créer. S'orienter procède de la construction de soi et ce processus se poursuit tout au long de la vie.

2 Les auteurs se donnent pour objectif d'élaborer un cadre théorique suffisamment général pour que l'on puisse y adosser, dans les divers contextes nationaux, des pratiques innovantes du conseil conçues comme une aide à la construction de soi. En contrepoint de ces principes généraux, nous nous proposons quant à nous de réfléchir aux conditions locales de leur application dans les pratiques françaises auprès d'adolescents. La spécificité de notre système scolaire - importance de l'orientation scolaire et étanchéité des filières - fait que les choix d'orientation des adolescents français sont à la fois précoces et déterminants pour l'insertion ultérieure. C'est dans le conseil auprès des adolescents que le modèle proposé par Savickas et al. peut donc trouver sa pleine justification mais aussi ses limites : il y semble pertinent parce que le conseil, tel qu'il est défini par les auteurs, a pour objectifs la réflexion sur soi, 
l'apprentissage de la distance critique et l'autonomisation, ce qui semble essentiel pour le développement personnel de l'adolescent face aux défis des choix et de l'insertion. Mais on peut supposer également que la sollicitation de la réflexion sur soi et sur ses «formes identitaires » de la part du consultant trouve ses limites lorsqu'il s'agit de travailler avec des adolescents jeunes, des collégiens par exemple, les possibilités de réflexion n'apparaissant que progressivement dans le développement.

Notre réflexion s'organise en trois points: le premier point est une discussion théorique des concepts de constructivisme et de réflexivité. Le deuxième point est empirique et présente succinctement les constats de recherches longitudinales que nous avons effectuées sur l'évolution des intentions d'orientation de collégiens, de lycéens et d'étudiants. Le troisième point est méthodologique : il s'agit de l'analyse d'un extrait d'entretien constructiviste de conseil.

\section{Proximités conceptuelles}

\section{Constructionnisme, constructivisme}

4 L'article de Savickas et al. se réfère à plusieurs reprises à l'épistémologie du constructionnisme social, courant pour lequel l'ensemble des productions humaines sociales - valeurs, représentations sociales, traditions, recherches scientifiques, idéologies et pratiques - sont construites par les interlocutions et les échanges avec autrui et par les interactions avec les contextes (Gergen, 1999/2001). En ce qui concerne l'orientation, on peut donc classer dans cette rubrique les représentations sociales des professions (Jodelet, 1994), les théories spontanées de l'orientation et les stéréotypes que partagent largement les individus selon leur groupe d'appartenance (Guichard \& Cassar, 1998) ainsi que les modes, les modèles et les mythes professionnels que partagent les adolescents en fonction de leur classe d'âge (Dumora, 2000).

Cependant, lorsqu'il s'agit de définir le travail individuel avec le consultant, ne serait-il pas plus approprié de se référer au constructivisme social? Gergen distingue en effet constructionnisme social et constructivisme social. Celui-ci, tout en affirmant que la réalité subjective est multiple et se construit dans les différents contextes sociaux de la vie, met l'accent sur le sujet individuel conçu comme interprétant et construisant sa propre réalité : le centre de gravité de la construction de soi réside dans la capacité du sujet à la réflexivité sur soi. Un des auteurs les plus représentatifs du socioconstructivisme est Bruner pour qui le self est à la fois le produit de l'histoire et de l'existence conjointe avec autrui et celui de la réflexivité humaine. Le self n'est pas intuitivement évident, c'est une construction réflexive qui procède autant de l'intérieur - de la mémoire, des sentiments, des croyances, de la subjectivité - que de l'extérieur des attentes d'autrui que nous reconnaissons très tôt dans nos différents contextes de vie. Retenons aussi de Bruner l'idée que le self est un récit autobiographique permanent qui fabrique de la signification de notre expérience et de notre vie au sein d'une culture (Bruner, 1986/2000) et que nous pouvons par le récit que nous en faisons reconceptualiser le passé et par notre capacité à trouver des alternatives fabriquer d'autres manières d'être, d'agir ou de lutter. La pratique du récit se nourrit évidemment dans les modèles culturels de la personnalité d'une société donnée, mais ces modèles ne sont jamais catégoriques et laissent une marge de manœuvre à l'individu pour se construire comme unique et différent d'autrui (Bruner, 2002). 
6 Si nous faisons cette remarque, c'est que l'argument central de l'article de Savickas et al. consiste à considérer que le consultant - le sujet, donc - a une prise sur ce processus de construction de soi, une possibilité d'utiliser cette marge de manœuvre dont parle Bruner. Ceci constitue même le noyau dur de l'argumentation : la première marche théorique est que la construction de soi du sujet est un processus interminable de construction et de reconstruction de ses propres réalités subjectives et multiples. De cette première affirmation découle, sur le plan des pratiques, la préconisation d'un type de conseil qui vise à amener le consultant à prendre conscience des contenus mentaux de ses intentions d'orientation, c'est-à-dire, selon le modèle de Guichard (2004, 2008) largement repris dans l'article de référence, du système de «formes identitaires » dans lesquelles le consultant s'imagine et se définit. Cette prise de conscience suppose un processus de réflexivité : il s'agit de solliciter chez le consultant, l'adolescent par exemple, la capacité à se décentrer et à observer, comme de l'extérieur, les contenus, c'est-à-dire les représentations de soi, présentes, passées ou anticipées, celles que l'adolescent a de lui-même, celles de l'adolescent qu'il est, qu'il aimerait être, ou du professionnel qu'il rêve d'être ou qu'il rejette, etc. Pour le dire avec les mots de Touraine (1995), sociologue théoricien du sujet, il s'agit pour le consultant d'une véritable interrogation identitaire, d'une prise de conscience des actes, d'une activité critique et d'une recherche de distance à soi qui permet de "se décaler pour s'affranchir». C'est par cette activité que le sujet peut prendre conscience des déterminismes et des influences extérieures, de l'évidence des idées toutes faites, des stéréotypes, des conceptions, injonctions et formes identitaires que projette l'entourage sur le sujet.

\section{Modèle éducatif, modèle constructiviste}

7 Cette argumentation (de la description du processus à son « activation " par le travail du conseil) est proche de celle qu'ont développée, il y a plus de trente ans, les auteurs de l'Activation du développement vocationnel et personnel (Pelletier, Noiseux \& Bujold, 1974), ensemble théorique et pratique qui a largement inspiré les pratiques de l'orientation en France au cours des trente dernières années. Le modèle canadien s'affirme déjà comme constructiviste: "la tâche éducative [...] s'inscrit dans une conception constructiviste du choix " (Pelletier \& Dumora, 1984, p. 29) et c'est aussi pour répondre aux besoins d'une société marquée dans les années 1970 et 1980 par le développement technologique, par la transformation des emplois et leur imprévisibilité, que les auteurs souhaitent substituer une pratique éducative aux pratiques psychométriques. La troisième révolution industrielle, selon le terme de Cohen (2006), celle des micro-processeurs et de l'Internet, ne fait donc qu'amplifier l'inadéquation des modèles traditionnels sans changer la nature du problème.

8 En résumé, l'activation selon Pelletier et al. consiste 1) à faire vivre des expériences au consultant, notamment à l'adolescent, 2) à faire «traiter cognitivement» ces expériences, c'est-à-dire à faire prendre conscience de leurs significations subjectives en proposant un travail de réflexion et de conceptualisation (formulation de comparaisons, d'analogies et de symboles, etc.) sur les émotions et les affects éprouvés et sur les images de soi ; enfin, 3) à amener le sujet à exercer un sens critique par rapport à ses manières de voir habituelles pour " qu'il se déconditionne de plus en plus des stéréotypes, des préjugés, des idées reçues» (Pelletier et al., 1974, p. 82). Reconnaissons que ces principes sont respectivement proches de la centration sur soi 
et sur son expérience, de la réflexivité et du décalage préconisés dans l'approche constructiviste.

Des différences essentielles distinguent pourtant les deux modèles : la construction des intentions d'orientation s'ordonne, pour Pelletier et ses collaborateurs, selon une séquence vocationnelle de quatre phases, inspirée, via la théorie de Super, du problem solving (exploration-cristallisation-spécification-réalisation) et marquée par la prégnance à chaque phase d'un processus cognitif spécifique. Le postulat de l'éducabilité de ces processus a entrâné la construction de programmes éducatifs collectifs censés stimuler successivement les processus correspondants. De nombreux programmes adaptés au système scolaire français s'appliquent encore aujourd'hui, dans les collèges notamment. Le schéma de la séquence vocationnelle n'est pourtant pas sans ambiguïté quant à son statut théorique et à sa temporalité: est-ce un processus ontogénétique de développement vocationnel dans une temporalité étendue à la carrière ou à un cycle d'études ? Est-ce la structure récurrente de résolution d'un problème de prise de décision? La succession des stades qui la constituent n'est pas issue des acquis essentiels de la psychologie du développement dont on peut dire que le passage des contenus concrets à des contenus plus abstraits et plus complexes est le plus petit dénominateur commun (Piaget, 1975 ; Fisher, 1980 ; Lautrey, 2006 ; Lehalle, 2006). Ce modèle apparait davantage comme une fiction pédagogique, ou comme un modèle rationnel de choix, bien plus qu'un modèle psychologique.

C'est pour cette raison qu'à l'idée d'un ordre hiérarchique de tâches, Savickas et al., à la suite de Van Esbroeck, Tibos, \& Zaman (2005), substituent l'idée de mini-cycles récurrents. Avec ses concepts d'activités plutôt que de tâches, de mini-cycles plutôt que de stades hiérarchiques, enfin de système, de boucles et d'interconnexions plutôt que de séquences ordonnées, ce modèle s'avère plus compatible avec la réalité psychologique des individus, souvent désordonnée et mouvante. Il peut constituer d'autre part un cadre beaucoup plus pertinent face aux trajectoires professionnelles d'aujourd'hui, imprévisibles et instables.

Une autre différence entre les deux modèles réside dans le rapport entre le sujet et le contexte. Le modèle explicitement personnaliste et rogérien de Pelletier et al. le conçoit comme un rapport d'extériorité. Certes, les contraintes situationnelles de l'orientation ne sont pas niées et le sujet est censé rencontrer, voire même anticiper, l'impossibilité d'actualiser ses désirs professionnels et la nécessité de réaliser des compromis. Mais la limitation par les circonstances extérieures semble se situer en aval de la construction des préférences. En amont en revanche, le modèle n'évoque pas, même de façon très générale, en tous cas dans les ouvrages de 1974 et 1984, la question des déterminants sociaux et scolaires des représentations et des préférences professionnelles. Au contraire, le modèle de Savickas et al. est à la fois développemental, holistique et contextuel : le contexte de vie, les positions sociales et le sexe sont déterminants dans le processus de construction identitaire et par là même dans l'élaboration des représentations et des préférences professionnelles. L'argumentation théorique de l'article est d'ailleurs consonante avec les approches contextuelles du développement : l'approche écologique de Bronfenbrenner (1979) qui décrit l'interaction de l'individu avec les différents niveaux de contexte, du plus local au plus englobant, avec celle de Lerner (1978) dont le concept d'enchâssement signifie que le développement humain est affecté par les interactions entre l'individu et ces différents niveaux de contexte, enfin avec le contextualisme développemental de Vondracek et al. (1986) pour qui la 
relation individu-contexte est un processus adaptatif réciproque entre un sujet actif et en développement et des contextes eux-mêmes structurés, interconnectés et changeants.

12 Mais il faut bien dire que ces deux modèles, comme la psychologie de l'orientation en général, ne se sont pas approprié les connaissances les plus représentatives de la psychologie du développement, sinon de façon très globale comme dans la théorie de Super dans laquelle l'adolescence est brossée à grands traits. Il nous semble souhaitable, comme le prônent au demeurant Savickas et ses collaborateurs, de consolider les assises théoriques du modèle constructiviste par une connaissance aussi approfondie que possible des mécanismes psychologiques de l'évolution des intentions d'orientation à cette période. Une question majeure se pose en effet à propos de la métaréflexion : peut-on faire progresser le sujet dans ses capacités métaréflexives par la sollicitation de cette catégorie de pensée au cours des entretiens? Ces modèles auraient à gagner à s'appuyer sur la théorie des apprentissages métacognitifs de Vygotski, socioconstructiviste de premier plan et de première heure (1934/1997). Sa théorie des liens entre apprentissages et développement permet en effet de comprendre comment l'individu peut acquérir une connaissance assez claire de ses propres processus de raisonnement lorsqu'il est mis dans des conditions de réfléchir à un niveau d'interrogation et dans des directions qu'il n'est pas capable d'envisager seul: c'est ce que Vygotski nomme la zone de développement proximal. Ces termes désignent le progrès que le sujet peut réaliser dans sa réflexion lorsqu'il est aidé par un adulte. La réflexion à ce niveau produit des effets à long terme sur les ressources personnelles du sujet acteur. Dans une transposition au champ du conseil, transposition qu'il faudrait approfondir pour la rendre opératoire, on peut dire que le développement actuel marque ce qu'un individu est capable de maîtriser seul, le type et le niveau de fonctionnement qu'il est capable de mettre en œuvre de façon autonome lorsqu'il évoque et argumente ses intentions d'orientation. La zone de développement proximal est le progrès qu'il peut réaliser dans cette réflexion et métaréflexion lorsqu'il est aidé par le conseiller.

Dans un deuxième point, il nous semble pertinent de présenter une synthèse succincte de nos propres travaux de recherche en psychologie du développement parce qu'ils sont centrés sur l'évolution des intentions d'orientation à l'adolescence et parce que nous avons analysé précisément la métaréflexion adolescente dans ce domaine (Dumora, 1990, 2000 ; Dumora, Gontier, Lannegrand, Pujol \& Vonthron, 1997).

\section{L'évolution des intentions d'orientation à l'adolescence : recherches empiriques}

14 Dans chacune de ces recherches indépendantes qui constituent des observations en temps réel de l'évolution des intentions d'orientation et de leur argumentation, les entretiens sont réitérés chaque année du cycle d'études avec chaque sujet. Sont sollicitées par le canevas d'entretien l'expression des intentions d'orientation, celle des arguments des préférences émises, des probabilités de réussite estimées et des solutions alternatives entrevues. Les énoncés des sujets très jeunes ne sont que des réponses succinctes à ces questions et le protocole de retranscription pour ces sujets est superposable au canevas. Mais progressivement, les réponses sont enchevêtrées dans le discours, le sujet articulant, joignant et disjoignant les questions du chercheur, 
dans une structure personnelle qui n'est plus superposable aux catégories préalables. Le protocole de retranscription réorganise donc le matériau discursif, en le délinéarisant, pour l'ordonner en fonction des catégories construites à partir d'analyses textuelles et de comparaisons intra et interindividuelles.

\section{Le tableau développemental : étude des processus}

On voit dans le tableau 1 que, pour chaque catégorie de processus mentaux (les différentes colonnes), le progrès dans le temps (d'une ligne à l'autre, de haut en bas) fait évoluer la pensée des modalités les plus concrètes, les plus figurées et les plus simples, vers des représentations et des raisonnements de plus en plus abstraits et complexes.

Tableau 1/Table 1

\begin{tabular}{|l|l|l|}
\hline Représentations professionnelles & Réflexion comparative & Réflexion probabiliste \\
\hline Perception syncrétique & Tautologique & Magique \\
\hline Imagée : collections, scripts et scènes & Métaphorique & Prédictive \\
\hline Propositionnelle & Métonymique & Prévisionnelle \\
\hline Fonctionnelle & Tensionnelle & Stratégique \\
\hline
\end{tabular}

Évolution des processus psychologiques des intentions d'orientation

Evolution of psychological processes of vocational choices

Les représentations professionnelles: chez le jeune collégien, elles sont syncrétiques et focalisées sur ce qui se voit, sur les gestes professionnels et les situations les plus visibles, dans une compréhension du monde simpliste, manichéenne, schématique, réductrice. L'intention professionnelle est d'abord une simple participation fusionnelle ou une identification globale et syncrétique à un personnage professionnel familier ou médiatique. Ce n'est que tardivement que de très rares collégiens et plus fréquemment des lycéens parmi les plus âgés se détachent de cette prégnance par le raisonnement et le sens critique, rompant avec la pensée manichéenne et magique de l'enfance.

La réflexion comparative: elle constitue l'argumentation proprement dite des intentions, qu'il s'agisse de simples préférences, de choix affirmés, ou de projets élaborés. C'est un travail mental de mise en tension entre les représentations de soi et les représentations professionnelles. Chez le jeune adolescent, c'est un rapprochement maladroit, un raisonnement métaphorique - de façon globale, on voudrait «faire comme » ou «être comme»-, souvent d'une grande pauvreté sémantique: l'argumentation est plus atomisée qu'articulée et elle tourne court la plupart du temps lorsqu'on demande au sujet de s'expliquer. La projection sur le personnage professionnel se fait ensuite plus analytique - métonymique -, et l'argumentation délimite une sorte d'intersection de qualités communes, entre le soi et l'image du professionnel, comparaison qui se systématise progressivement. L'avènement progressif des capacités d'abstraction permet à l'adolescent plus âgé, lycéen ou étudiant, la perception de la complexité du soi, voire de ses contradictions, et des effets des contextes sur ses préférences ou ses 
intentions. Il devient alors capable d'analyser les émotions et les affects qu'il éprouve en les évoquant et de prendre ses distances par rapport à certaines motivations qu'il peut désigner comme imposées, comme stéréotypées ou comme immatures.

La réflexion probabiliste: c'est un travail de conceptualisation de l'incertitude par lequel les adolescents, confrontés par autrui à la question de leur avenir, tentent de se représenter et d'évaluer les contraintes et leurs atouts. La réflexion probabiliste progresse globalement de la certitude magique et de l'affirmation péremptoire chez les plus jeunes vers une confrontation de plus en plus organisée entre les compétences exigées par les professions ou les formations et les autoévaluations. L'élaboration des catégories mentales du possible, du probable et du réel donne la capacité à penser le doute, l'incertitude, l'aléatoire, la chance, le hasard de l'orientation. Se met en place aussi progressivement la capacité de raisonnement sur des critères, des scénarios possibles, sur des hiérarchies, sur des solutions de repli, sur des plans de secours.

Le tableau qui est un résumé des comparaisons interindividuelles se présente comme un tableau développemental avec ses progrès catégorisables en stades. Mais si on considère l'évolution des contenus et des processus mentaux chez un même individu (comparaisons intra-individuelles dans le temps), on observe des progressions, des régressions, des hésitations, des décisions subites ou même l'absence de préférences et de motivations, problèmes que rencontrent les conseillers d'orientation-psychologues dans leurs consultations. Les entretiens font en effet apparaître cette matière mentale toujours très compliquée, mélange de préférences ou de fictions professionnelles, de hiérarchies scolaires et d'évaluations de soi, d'intentions soudaines, d'illusions résistantes, d'évidences familiales, ou de "pannes» de motivation, voire de rejet. L'évolution des intentions d'orientation est un exemple d'un problème débattu en psychologie du développement: la psychologie du développement actuelle ne postule plus l'avènement au cours de l'adolescence d'une organisation abstraite commune l'abstraction - dont découlerait une synchronisation des acquisitions dans tous les domaines (Fisher, 1980) et les conquêtes cognitives ne s'effectuent pas indépendamment des domaines fonctionnels d'application. La compréhension des phénomènes sociaux et du fonctionnement de l'orientation constitue un de ces domaines d'application des capacités cognitives: or, le degré de visibilité des professions, la prégnance de certaines dans l'environnement, le degré de familiarité que les sujets peuvent avoir avec elles, la figurabilité de ces professions, c'est-à-dire leur capacité à générer des images mentales, ces différences d'accessibilité modulent la représentation que l'adolescent peut en construire et les activités réflexives qu'il peut exercer.

\section{Des mythes d'une classe d'âge à l'accommodation scolaire : étude des contenus des intentions}

20 Nos études montrent que l'imaginaire professionnel des jeunes collégiens est souvent un imaginaire sans imagination puisqu'il converge de façon grégaire vers les mythes d'une classe d'âge et l'adhésion stéréotypée à des figures, notamment médiatiques (Dumora, 1998). Malgré l'évolution des rôles sociaux des hommes et des femmes, l'imaginaire des filles reste ainsi longtemps focalisé sur les métiers de la séduction féminine, de l'enfance et de l'humanitaire. Le conformisme de ces fictions professionnelles cède toutefois la place à un deuxième registre, stratégique et scolaire, 
qui se met en place lors de la découverte du sérieux de la question d'orientation, de son enjeu et de la hiérarchie des formations. Car l'école procède à une sorte d'assignation de place et tout adolescent découvre, au fur et à mesure de la scolarité, en collège d'abord puis au lycée, que son orientation va dépendre de ses compétences, mesurées le plus souvent à travers des performances scolaires, plus ou moins étroitement bien sûr, et plus ou moins précocement. L'adolescent découvre ainsi que son goût, ses préférences, ses intérêts, sa personnalité, ses penchants, ne jouent que dans une certaine zone qui, elle, est déterminée d'abord par ses performances. Alors que jusqu'ici tout lui apparaissait possible, il prend conscience de ce qui est probable pour lui. Ce rétrécissement des possibles en un espace restreint de probables est une première déconvenue pour beaucoup.

21 La comparaison des suivis individuels et des modes de résolution face à la frustration et à l'échec a permis de construire une typologie des trajectoires des adolescents. Quatre types apparaissent :

22 - le discours lisse de l'excellence scolaire des adolescents chez qui peuvent coexister des ambitions scolaires précises et prestigieuses et des intentions professionnelles floues et changeantes, parce que différées ;

23 - le discours de l'illusion d'adolescents qui maintiennent magiquement jusqu'aux échéances une croyance illusoire en la réussite malgré les contraintes perçues et les exigences qui sont pourtant évoquées par certains. Ils juxtaposent des fins improbables, et dont ils savent désormais qu'elles sont improbables, et des constats d'échec actuel, dissonance qui ne se résout souvent que par une capitulation pure et simple des préférences au moment des échéances de l'orientation ;

24 - le discours de la résignation: il accompagne une suite de choix de moins en moins valorisés et de solutions de moindre mal. Les adolescents de cette catégorie, eux, apparaissent très vite conscients des renoncements à effectuer. Certains d'entre eux, parce qu'ils ne peuvent pas reconstruire une motivation ni construire le sens de leur expérience scolaire, sont amers et désabusés ;

25 - le discours de la rationalisation est le plus fréquent: la dissonance introduite par la confrontation entre le désirable et le probable conduit les adolescents à ajuster leurs préférences à leur niveau scolaire, donc à renoncer à des accomplissements plus prestigieux à leurs yeux. Leur argumentation est une sorte d'accommodation cognitive. Ils s'accommodent, au sens piagétien du terme, à l'assignation qui leur est faite. Ce processus de rationalisation est un processus très actif dans la construction des intentions d'avenir des adolescents (Dumora \& Lannegrand-Willems, 1999). Plus salutaire du point de vue psychologique que la résignation ou que l'illusion, qui sont véritablement inopérantes parce que ce sont des retraits, la rationalisation permet d'accepter les situations qui sont imposées. Processus à double tranchant dont on peut voir l'aspect adaptatif dans la fonction restauratrice et bénéfique pour le sujet lorsqu'il est placé de fait dans certaines conditions d'orientation ou d'emploi, mais il comporte le risque d'amener certains sujets, déjà prompts à renoncer, à la soumission. On pense ici plus particulièrement aux adolescents peu soutenus par leurs familles lorsqu'elles sont défavorisées ou trop éloignées des savoirs scolaires ou peu familières avec les stratégies efficaces.

26 C'est pour les trois dernières catégories de sujets (illusion, résignation et rationalisation) qu'on saisit le mieux la portée d'un conseil qui ferait naitre chez ces 
sujets d'une part la capacité de se décaler par rapport aux dévaluations de soi qui leur sont ainsi infligées, de les relativiser en les situant dans un système de significations institutionnelles. Mais il s'agit aussi, d'autre part, de prendre conscience des façons qu'ils ont de traiter le problème d'orientation, les uns s'enfermant dans l'impasse de l'illusion, les autres dans le retrait, les derniers dans une acceptation qui peut être coûteuse.

\section{La capacité métaréflexive des adolescents}

Ce travail de prise de conscience peut apparaitre spontanément dans les entretiens, qu'ils soient de recherche ou de consultation. Notre recherche a ainsi mis en évidence une catégorie d'énoncés non directement suscitée par les questions du canevas, ni classables après-coup dans les catégories dégagées par l'analyse : nous avons appelé métaréflexion cette catégorie d'énoncés spontanés parce qu'elle regroupait des jugements auto-critiques à la fois introspectifs et rétrospectifs sur les préférences émises ou sur leur argumentation, des remarques sur les contradictions que les sujets relevaient dans leurs propres discours, des critiques des discours d'autrui, discours familiaux surtout sur l'orientation, des qualifications ou disqualifications de préférences émises antérieurement, etc., soit un ensemble de discours sur le discours qui nous semblait justifier cette catégorisation de métaréflexion. Nous pensons que la métaréflexion que nous avons observée est bien de l'ordre de celle que préconisent le modèle de Guichard et l'article de Savickas et al.

Que pouvons-nous en dire? L'analyse de ces énoncés spontanés montre qu'ils augmentent au fur et à mesure de l'avancée en âge et qu'ils se différencient progressivement comme le montre le tableau 2. Chez les plus jeunes adolescents, la métaréflexion, lorsqu'elle existe, ne porte que sur les contenus professionnels: au cours de l'entretien, ils les désignent parfois comme une conviction personnelle, et précisent l'accord ou le désaccord avec les conseils familiaux. Rétrospectivement, lorsqu'ils évoquent leurs préférences passées, ils peuvent les désigner comme des croyances immatures, comme des rêves ou comme des effets d'une adhésion révolue aux goûts des autres. Plus tardivement, chez le collégien de $3^{e}$ ou chez le lycéen, la métaréflexion distingue les catégories du possible, du probable et de l'incertain et argumente cette classification. Plus tardivement encore, la métaréflexion peut aussi porter sur les processus de l'élaboration des intentions d'orientation : les lycéens et les étudiants peuvent évoquer l'influence des personnes de leur entourage, leurs projets pour eux, leurs stéréotypes, leurs critères, ils peuvent analyser les termes des conflits à propos de l'orientation. Ils sont capables de comparer les implications quant à leur vie future et à leur identité des différentes possibilités d'orientation entre lesquelles ils hésitent. L'exemple de Marie que l'on examinera plus loin éclaire ce point et montre en quoi cette auto-réflexivité constitue une avancée de la construction de soi.

Tableau 2/Table 2

Métaréflexion

Appréciation manichéenne des contenus imagés

Reconnaissance des nuances et de la pluralité des perspectives 
Hiérarchisation et évaluation des préférences

Explication du statut des préférences : probables, possibles, rêves, illusions

Réflexion sur les processus d'élaboration des préférences: influences subies, modes, effets d'appartenance, stéréotypes

Évolution de la métaréflexion

Evolution of metareflexion

\section{Extrait d'entretien : énoncés métaréflexifs}

Rappelons que dans nos recherches passées, les discours métaréflexifs sont spontanés, le chercheur ne visant aucunement à les solliciter. En revanche, dans l'entretien avec Marie, présenté ci-dessous, le chercheur sollicite activement la centration du sujet sur ses propres discours, cet entretien ayant été réalisé dans le cadre des travaux actuels sur la réflexivité dans l'entretien de conseil constructiviste (Guichard \& Dumora, 2008). Cet extrait montre les diverses facettes de la réflexivité. Marie est une lycéenne inquiète pour son choix d'études supérieures. Sa réflexivité porte spontanément, puis systématiquement avec l'aide du chercheur, sur les contenus comparés de ses préférences d'études et de profession, mais surtout sur les processus cognitifs et affectifs qui les influencent. Méthodologiquement, la sollicitation du chercheur prend la forme soit de relances ou de demandes neutres d'approfondissement sur les thèmes abordés par la jeune fille, soit des reformulations d'énoncés choisis incitant à élaborer différemment les arguments évoqués et à opérer de nouveaux rapprochements.

Marie évoque les rapports avec les personnes de son entourage et compare les conseils maternels et paternels : au conseil sage, statique et traditionnellement féminin de la mère, elle oppose l'ouverture, l'ambition et la plasticité inhérentes au conseil paternel. Elle résume son désarroi à plusieurs niveaux dans une métaréflexion très aiguisée : désarroi entre son refus de l'idéal féminin de sa mère, précis et sécurisant, mais insatisfaisant pour elle, et l'attrait d'études dépaysantes, mais imprécises et incertaines du conseil paternel, désarroi face à sa propre indécision, dont elle mesure l'intensité et repère les différentes strates: entre les types d'études, entre les carrières possibles, entre la sagesse d'études balisées ou l'attrait du risque et de l'ouverture, entre un engagement immédiat ou différé, entre une installation locale aisée ou un dépaysement lointain, entre un modèle professionnel féminin ou masculin, etc.

Alors, entre ma mère qui me dit de faire orthodontiste [...] pour les femmes, on peut gérer ses horaires, pas d'urgences et c'est un métier où on gagne bien sa vie, etc. [...] mon père, lui, il me dit: une petite école d'ingénieur, parce que je sais bien que je ne peux pas faire une grande, bon, alors il me fait miroiter, tu iras passer un an aux États-Unis pour te former, faire un MBA ou autre, et moi dans tout ça ? [...] mon grand-père, il me dit: si tu veux faire médecine, tu peux passer par Santé navale, c'est un excellent moyen parce qu'il paraît qu'on prépare très bien le concours de $1^{\text {re }}$ année.

Mais alors moi dans tout ça, l'idéal féminin de ma mère? Je sais ce que ça me fait quand elle me dit ça : orthodontiste mais c'est comme institutrice pour elle, tous les soirs à la maison, ça m'horripile à l'avance, et si ça m'horripile, je ne peux même pas regarder ça en face. C'est ça, quoi, c'est la façon dont elle veut que je fasse finalement comme elle, elle est pharmacienne! Mon grand-père: assurer le 
concours de médecine, oui, c'est la sagesse finalement. Je sais plus quoi faire, je me sens coincée par les gens qui me le disent. Ma mère c'est clair. Avec mon père, je peux voir ce que ça veut dire sans me faire avoir. Mon grand-père, je comprends bien et j'essaie de voir. Mais moi, mon idéal, il n'est pas tellement là pour le moment! Moi, ce serait plutôt genre année tranquille, voyager autour du monde, je ne pourrai pas concilier tout de toutes façons, je me vois mieux finalement en médecine et après je verrai, et même une spécialisation aux États-Unis pourquoi pas. Mais orthodontiste, j'en ai eu plein la bouche pendant cinq ans, ma mère ça la fait rêver pas moi [...]. Mon père, bon, l'international et l'ouverture, les facs américaines, ça assure, là, je rêve plutôt, alors si je compare, là ça m'horripile pas, ça. Ou plutôt, ça me fait voir encore plus orthodontiste [...] enfin, c'est pas orthodontiste, c'est les arguments de ma mère, quoi. Mon grand-père il se propose même de me préparer au concours de Santé navale. Je tire au sort ou quoi entre les trois?

Comment choisir? D'un côté, j'adore les maths et je suis bonne, donc je me vois faire des études genre ingénieur, classe prépa. Mais le métier lui-même, ça me dit pas tellement. Bon, médecine, c'est le contraire : huit ans pour des études qui ne me plaisent pas trop, biolo, biolo, biolo [...] mais le métier, c'est vraiment ce que je sais que je veux faire. Médecine pour moi, il y a pas mieux, c'est ça, oui, vraiment. Mais en même temps abandonner les maths, qu'est-ce que je trouve dommage !

31 La réflexion de Marie ne manque ni de saveur ni de distance critique et elle évoque même provisoirement une année de voyage pour signifier, à elle-même et aux autres, qu'il lui faudrait bien ce temps-là et ce dépaysement-là pour prendre des distances par rapport aux influences parentales et pour résoudre son problème de choix qu'elle finit par décliner comme un problème d'indécision identitaire. C'est sur ce travail de réflexion identitaire que se poursuivra l'entretien pour une "délibération" personnelle qui pourra conduire Marie à opter pour une décision au détriment de l'autre ou à l'élaboration possible d'un compromis satisfaisant, tant sur le plan du choix d'études que de l'engagement identitaire qui le sous-tend. L'exemple de Marie illustre remarquablement ce que peut être une "cartographie des formes identitaires ». Elle évoque les rapports avec les personnes de l'entourage, elle repère, compare et critique leurs critères, leurs stéréotypes, leurs modèles implicites, leurs raisonnements et leurs contradictions. Elle formule ses propres hésitations, ses critères, ses difficultés à ordonner et évaluer les influences extérieures, etc. Si elle n'a pas les termes savants pour désigner ces discours parentaux, elle en fait une description analytique, riche et nuancée. Dans le cas de Marie, le conseil n'appelle finalement qu'un accompagnement de l'examen des coûts et des avantages comparés des solutions envisagées.

\section{Conclusion}

Les praticiens du conseil ne manqueront pas d'observer qu'un tel niveau de métaréflexion est bien rare, y compris chez des étudiants plus âgés et que Marie est dans un environnement familial informé et stimulant, même si les " projets » des uns et des autres dans la famille semblent difficilement conciliables pour la jeune fille. Mais dans des populations socialement défavorisées ou chez des élèves en grande difficulté scolaire stigmatisés par des évaluations négatives, l'absence de motivation, voire l'absence d'espoir, génère les plus grandes difficultés du conseil. C'est pourtant lorsqu'il s'agit de travailler avec les adolescents peu ou pas stimulés, peu ou pas informés par leur environnement familial, que le conseil auprès d'adolescents prend tout son sens psychologique et trouve toute sa justification. La sollicitation de la centration sur soi - 
pour que le sujet «se décale " par rapport à ses propres discours, ses récits de soi, ses perceptions ou ses images de lui-même - peut l'amener à prendre conscience que la façon dont il se perçoit et dont il perçoit le monde, celui de l'école ou celui des professions par exemple, est tributaire du contexte dans lequel il se situe et des discours qui y sont tenus, et donc à relativiser cette perception. Ceci est d'autant plus important que le point de vue de l'adolescent risque de se rigidifier dans le manichéisme le plus appauvrissant lorsqu'il est en difficulté. Et ceci souvent faute de mots, d'analyse, de capacités à relativiser et de situations d'écoute par d'autres. Le conseiller peut justement lui apporter d'autres mots, des capacités d'analyse et des moments d'écoute. Il peut l'amener à repérer, expliciter et nommer différentes contraintes personnelles ou contextuelles (problèmes financiers, handicaps, préjugés, stéréotypes, croyances, modes, désirabilité sociale) et à prendre conscience de la façon dont ces contraintes peuvent limiter la façon dont il peut ou pourrait se projeter dans l'avenir. L'adolescent peut comprendre qu'une profession donnée - ou un comportement, ou une décision, ou une orientation, ou encore une expérience sociale peut être considérée différemment par sa famille, par ses pairs et par lui-même selon les moments. Pour élargir son répertoire de mots et de descriptions de soi et pour multiplier les points de vue possibles, l'ensemble des méthodes élaborées par la psychologie de l'orientation depuis des décennies - outils évaluatifs, méthodes de discussion en groupes, séquences d'éducation à l'orientation, etc. - peuvent être utilisées dans une perspective constructiviste. Il s'agit d'amener l'adolescent à prendre conscience de ce qu'il privilégie dans les réponses qu'il donne, ou dans sa façon d'envisager le futur. Autrement dit, il ne s'agit plus seulement de trouver du sens à ses expériences et d'en faire émerger une intention d'orientation comme si ces choses allaient de soi - on risque fort alors de rester dans une logique reproductive -, mais bien de prendre vis-à-vis d'elles une position et une distance critiques pour une logique émancipative : on pourrait presque dire qu'une position constructiviste suppose aussi, dans certains cas, un certain travail de déconstruction...

\section{BIBLIOGRAPHIE}

- Bronfenbrenner, U. (1979). The ecology of human development. Cambridge, Mass: Harvard University Press.

- Bruner, J. (1986/2000). Culture et modes de pensée. Paris : Retz.

- Bruner, J. (2002). Pourquoi nous racontons-nous des histoires? Paris : Retz.

- Cohen, D. (2006). Trois leçons sur la société post-industrielle. Paris : Seuil-La République des Idées.

- Dumora, B. (1990). La dynamique vocationnelle chez les adolescents de collège : continuité et ruptures. L'Orientation Scolaire et Professionnelle, 19(2), 111-127.

- Dumora, B. (1998). L'imaginaire professionnel des jeunes adolescents. Carriérologie, 7(1 \& 2), 3-32. 
- Dumora, B. (2000). Les intentions d'orientation. Aspects développementaux et psychosociaux. (HDR). Paris : INETOP-CNAM.

- Dumora, B., Gontier, C., Lannegrand, L., Pujol, J.-C., \& Vonthron, A.-M. (1997). Expérience étudiante et déterminismes scolaires. L'Orientation Scolaire et Professionnelle, 26(3), 389-414.

- Dumora, B., \& Lannegrand-Willems, L. (1999). Le processus de rationalisation en psychologie de l'orientation. L'Orientation Scolaire et Professionnelle, 28(1), 3-29.

- Fischer, K. W. (1980). A theory of cognitive development: The control and construction of hierarchies of skills. Psychological Review, 87, 477-531.

- Gergen, K. G. (1999/2001). Le constructionisme social. Une introduction. Paris : Delachaux et Niestlé

- Guichard, J., \& Cassar, O. (1998). Social fields, habitus and cognitive schemes. Study stream and the categorization of occupations. Revue internationale de Psychologie sociale, 11, 123-145.

- Guichard, J. (2004). Se faire soi. L'Orientation Scolaire et Professionnelle, 33(4), 499-533.

- Guichard, J. (2008). Proposition d'un schéma d'entretien constructiviste de conseil en orientation (life designing counseling) pour des adolescents ou de jeunes adultes. L'Orientation Scolaire et Professionnelle, 3(37), 313-340.

- Guichard, J., \& Dumora, B. (2008). A constructivist approach to ethically grounded vocational development interventions for young people. In J. A. Athanasou \& R. Van Esbroeck (Eds.), International Handbook of Career Guidance, 187-208. Springer Science.

- Jodelet, D. (1994). Représentations sociales : un domaine en expansion. In D. Jodelet, Les représentations sociales. Paris : Presses universitaires de France.

- Lautrey, J. (2006). Les théories néo-piagétiennes. In S. Ionescu \& A. Blanchet (éd.), Nouveau cours de psychologie. Psychologie du développement et psychologie différentielle (pp. 78-102; vol. coordonné par J. Lautrey). Paris : Presses universitaires de France.

- Lehalle, H. (2006). Cognitive development in adolescence: Thinking freed from concrete constraints. In S. Jackson \& L. Goossens (Eds.), Handbook of adolescent development (pp. 71-89). Hove and New York: Psychology Press.

- Lerner, R. M. (1978). Nature, nurture and dynamic interactionism. Human Development, 21(1), $1-20$.

- Pelletier, D., Noiseux, G., \& Bujold, C. (1974). Développement vocationnel et croissance personnelle. Montréal : Mac Graw-Hill.

- Pelletier, D., \& Dumora, B. (1984). Fondements et postulats pour une approche éducative. In D. Pelletier \& R. Bujold, Pour une conception éducative en orientation. Chicoutimi : Gaëtan Morin.

- Piaget, J. (1975). L'équilibration des structures cognitives. Paris : Presses universitaires de France.

- Touraine, A. (1995). La formation du sujet. In F. Dubet \& M. Wieviorka (dir.), Penser le sujet. Autour de l'œuvre d'Alain Touraine. Colloque de Cerisy. Paris : Fayard.

- Van Esbroeck, R., Tibos, K., \& Zaman, M. (2005). A dynamic model of career choice development. International Journal for Educational and Vocational Guidance, 5, 5-18.

- Vondracek, F. W., Lerner, R. M., \& Schulenberg, J. E. (1986). Career development: A life span developmental approach. Hillsdale, NJ: Lawrence Erlbaum.

- Vygotski, L. (1934/1997). Pensée et langage. Paris : La dispute/SNEDIT. 


\section{RÉSUMÉS}

Le premier point de cette contribution est une discussion des concepts de constructivisme, de constructionnisme et de réflexivité. Le deuxième point est une présentation de recherches longitudinales sur les contenus des préférences d'orientation à l'adolescence et sur les processus mentaux de l'élaboration de ces intentions d'orientation. À titre d'exemple, l'analyse d'un extrait d'entretien constructiviste de conseil montre ce que peut être la sollicitation de la réflexivité et sur quels contenus et processus mentaux elle peut porter.

The first point of this article is a discussion about the concepts of constructivism, constructionnism and reflexivity. The second point is a presentation of longitudinal studies about contents of vocational intentions during adolescence and psychological processes of their elaboration. As an example, a brief analysis of a counselling interview shows what reflexivity is and what are its contents and psychological processes.

\section{INDEX}

Mots-clés : Adolescence, Constructionnisme, Constructivisme, Modèle éducatif, Réflexivité Keywords : Adolescence, Constructionnism, Constructivism, Educative model, Reflexivity

\section{AUTEUR}

\section{BERNADETTE DUMORA}

Bernadette Dumora est chercheuse associée à l'équipe de Psychologie de l'orientation du Laboratoire CRTD (EA 4132), INETOP-CNAM. Thèmes de recherche : intentions d'orientation chez les adolescents et les jeunes adultes, réflexion comparative, réflexion probabiliste, métaréflexion, processus de rationalisation. Contact : Laboratoire CRTD - 41 rue Gay-Lussac - 75005 Paris.

Courriel : bdumora@neuf.fr 